\title{
Identificação de sítios de reprodução de Aedes aegypti com aeronave remotamente pilotada (ARP)
}

\author{
Matheus Antonio PEREIRA ${ }^{1 *}$, Normandes Matos da SILVA ${ }^{1}$ Domingos Sávio BARBOSA ${ }^{1}$, \\ Dhonatan Diego PESSI ${ }^{2}$, Antonio Pancracio de SOUZA³ ${ }^{3}$ Antonio Conceição PARANHOS FILHO²
}

\author{
${ }^{1}$ NUPEC, Universidade Federal de Rondonópolis, Rondonópolis, MT, Brasil. \\ 2 LabGIS, Universidade Federal de Mato Grosso do Sul, Campo Grande, MS, Brasil. \\ ${ }^{3}$ Educação em Saúde, Universidade Federal de Mato Grosso do Sul, Campo Grande, MS, Brasil. \\ *E-mail: matheuseng.pereira@gmail.com \\ (ORCID: 0000-0002-0545-5587; 0000-0002-4631-9725; 0000-0001-6793-0956; 0000-0003-0781-785X; \\ 0000-0003-2544-1120; 0000-0002-9838-5337)
}

Recebido em 06/04/2021; Aceito em 31/08/2021; Publicado em 20/09/2021.

\begin{abstract}
RESUMO: Um drone e seus complementos de voo são denominados Sistema de Aeronave Remotamente Pilotada (RPAS - Remotely Piloted Aircraft System), sendo uma ferramenta com ampla gama de aplicações em diversas áreas. A pesquisa prospectou novas possibilidades de uso de RPAS com enfoque no diagnóstico e monitoramento de locais de reprodução de Aedes aegypti. Para isso, objetos considerados como potenciais criadouros de larvas de mosquito foram distribuídos em ambientes que permitiam maior ou menor detecção visual dos alvos (embalagens/recipientes) em quatro ambientes: solo coberto com gramínea seca, solo exposto, solo coberto com gramínea de porte baixo e solo coberto com gramínea de porte alto. Foi utilizado RPAS, Phantom 4 Pro com dispositivo móvel e o programa nativo da RPA para os voos. Sobrevoamos alvos para registro fotográfico em quatro alturas do solo $(20 \mathrm{~m}, 30 \mathrm{~m}, 60 \mathrm{~m}$ e $80 \mathrm{~m})$. A detecção visual dos alvos foi realizada por um grupo de 10 pessoas denominado júri. O Júri aferiu a maior ou menor probabilidade de detecção de alvos, em função de três variáveis: tipo de alvo, tipo de ambiente e altura de tomada da fotografia aérea. Fotografias obtidas a 30 metros de altura representaram o maior número de alvos identificados (30\% dos alvos). Os alvos mais identificados foram pneu, garrafa PET, latas de cerveja e latas de tinta. Os menos identificados foram vasilhas plásticas coloridas e garrafas de cerveja. A pesquisa colaborou para o aperfeiçoamento de procedimentos operacionais de controle e combate a endemias e epidemias, que poderão identificar possíveis criadouros do mosquito por meio de RPA, monitorando áreas de difícil acesso que ofereçam risco a integridade física das pessoas.
\end{abstract}

Palavras-chave: drone; geotecnologias; arboviroses; dengue.

\section{Identification of reproduction sites of Aedes aegypti with remote pilot aircraft (ARP)}

\begin{abstract}
A drone and its flight accessories are called Remotely Piloted Aircraft System (RPAS - Remotely Piloted Aircraft System), being a tool with a wide range of applications in several areas. The research explored new possibilities for the use of RPAS with a focus on the diagnosis and monitoring of breeding sites for Aedes aegypti. For this, objects considered as potential breeding grounds for mosquito larvae were distributed in environments that allowed greater or lesser visual detection of targets (packages/containers) in four environments: soil covered with dry grass, exposed soil, soil covered with low grass. and soil covered with tall grass. Was used RPAS, Phantom 4 Pro with an Ipad Mini 4 mobile device and the DJI GO program for flights. We fly over targets for photographic recording at four heights from the ground $(20 \mathrm{~m}, 30 \mathrm{~m}, 60 \mathrm{~m}$ and $80 \mathrm{~m})$. The visual detection of the targets was carried out by a group of 10 people called a jury. The Jury assessed the greater or lesser probability of target detection, depending on three variables: type of target, type of environment and height of aerial photography. Photographs taken at a height of 30 meters represented the largest number of targets identified (30\% of the targets). The most identified targets were tires, pet bottles, cans of beer and cans of paint. The least identified were colored plastic canisters and beer bottles. The research helped to improve operational procedures for controlling and combating endemics and epidemics, which may identify possible mosquito breeding sites through RPA, monitoring areas of difficult access that pose a risk to people's physical integrity.
\end{abstract}

Keywords: drone; geotecnologies; arbovírus; dengue.

\section{INTRODUÇÃO}

A espécie Aedes aegypti (Diptera: Culicidae) popularmente conhecido como "mosquito da Dengue" é um importante vetor incriminado para arboviroses tais como Dengue, Chikungunya, Zika e Febre Amarela Urbana. De acordo com os dados do Ministério da Saúde, até a $32^{\mathrm{a}}$ semana epidemiológica do ano de 2020, o Brasil registrava 918.773 casos prováveis de Dengue, com taxa de incidência de 437,25 casos por 100 mil habitantes (BRASIL, 2020).

A expressão "focos de Dengue" refere-se aos habitats em que possivelmente o mosquito possa desenvolver parte de 
seu ciclo biológico. Fêmeas de $A$. albopictus, um importante vetor congenérico ao A. aegypti, apresentam preferências por determinados habitats para oviposição. Em linhas gerais, essas espécies são oportunistas em termos de habitat para oviposição, bastando haver um recipiente com água com pouca turbulência e lâmina de água que indique baixo risco de secagem (REISKIND; ZARRABI, 2012).

Deve-se fazer o controle de locais que servem de habitat artificial a oviposição, tais como resíduos sólidos do tipo garrafas, copos e pratos descartáveis, dentre outros, que são abundantes em terrenos baldios e canteiros na área urbana. O comportamento das fêmeas de $A$. aegypty frente aos locais candidatos a oviposição, constituem uma característica estratégica nos aspectos ecológicos, evolutivos e epidemiológicos. Em linhas gerais, existe uma tendência do aumento da oviposição proporcionalmente ao número de locais aptos à sua reprodução (ABREU et al., 2015).

As Aeronaves Remotamente Pilotadas (ARP) multirrotores, popularmente conhecidas como drones, possuem diversas aplicações, desde as mais simples como fazer imagens aéreas, onde é necessário que carregue apenas uma câmera, até procedimentos mais complexos como dispersão de sementes, para uso na silvicultura ou na recuperação de áreas degradadas. A palavra "drone" em tradução literal significa zangão, devido ao som produzido pelas suas hélices lembrar o som de abelhas, no caso de um equipamento do tipo multirotor, como os quadricópteros e hexacópteros, por exemplo. Um termo mais técnico para esse equipamento seria "VANT" ou Veículo Aéreo Não Tripulado, de caráter não recreativo. A Agência Nacional de Aviação Civil (ANAC) estabeleceu que o termo a ser adotado no Brasil é Sistema de Aeronave Remotamente Pilotada (RPAS) ou (Remotely-Piloted Aircraft System), que melhor descreve as funcionalidades da tecnologia (DECEA, 2019).

Para a pesquisa utilizou-se uma Aeronave Remotamente Pilotada, do tipo multirotor, modelo Phantom 4 Pro, da empresa DJI Phantom 4 Pro, pertencente à Universidade Federal de Rondonópolis. Essa RPA é um dos modelos mais populares no mundo. Outros fatores que corroboram para a escolha do mesmo é sua autonomia de voo, por volta de 20 minutos, sua portabilidade e agilidade operacional no registro de fotofilmagem. O fato de o equipamento ser um dos RPA mais populares no mercado, facilita replicar essa metodologia em outros lugares e difundir essa funcionalidade para a identificação, controle e combate de possíveis focos de Dengue.

Foi investigado o potencial de Aeronaves Remotamente Pilotadas (RPA) para a detecção e caracterização de objetos e locais em espaço urbano que podem ser focos de larvas de mosquitos. Foram identificados alguns parâmetros importantes para viabilizar a criação de um procedimento operacional padrão (POP) para a realização de voos com RPA, com o propósito de detectar de forma ágil criadouros de Aedes aegypti, contribuindo com órgãos de vigilância sanitária que fazem o controle de endemias e epidemias.

\section{MATERIAL E MÉTODOS}

\section{1. Área de estudo}

O experimento foi conduzido na área experimental (Figura 1) da Universidade Federal de Rondonópolis. O município de Rondonópolis está localizado na região Sudeste do estado de Mato Grosso, a 210 km da capital, Cuiabá. Possui uma população de aproximadamente 240 mil habitantes segundo estimativas do IBGE (IBGE, 2021). O clima é tropical com uma estação quente-úmida entre outubro e março e uma estação fria-seca entre abril e setembro (Aw no sistema de classificação de Köppen). A temperatura média anual é de $24^{\circ} \mathrm{C}$ a $26^{\circ} \mathrm{C}$, e a precipitação média anual é de 1000-1600 mm (ALVES., 2020). A vegetação Cerrado é mais presente, composto por Cerradão, Cerrados, Formação Savânica associada a vertentes e Floresta Estacional semidecídua com composição florística típica e com diferentes graus de caducifólia na estação seca (PESSI; LOVERDE-OLIVEIRA, 2019).
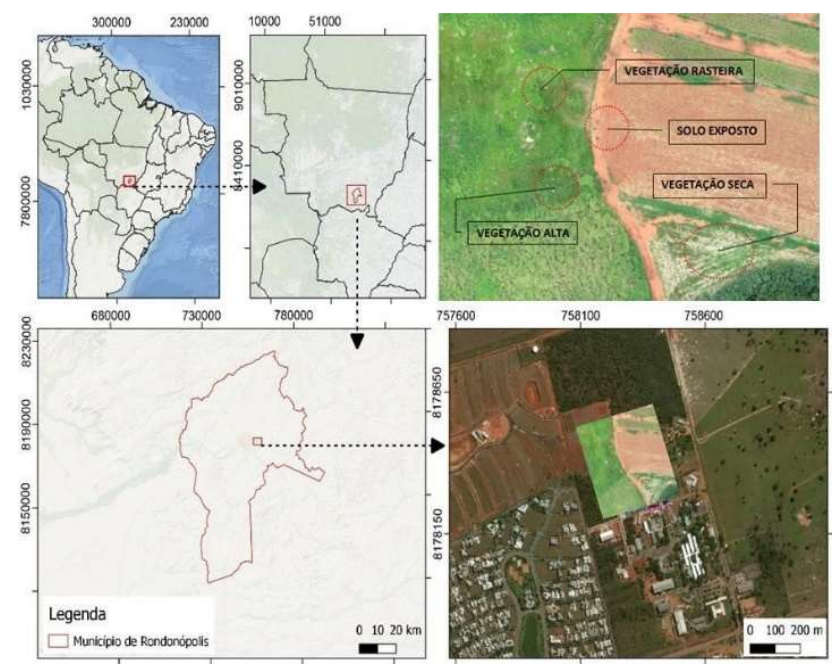

Figura 1. Área experimental localizada na Universidade Federal de Rondonópolis. Fonte dos vetores; IBGE (2019); imagens de satélite, Google (2019).

Figure 1. Experimental area located at the Federal University of Rondonópolis. Source vector data; IBGE (2019); satellite images, Google (2019).

\subsection{Alvos}

Houve a seleção dos alvos (objetos que podem armazenar água) com itens que estão mais presentes em campanhas de conscientização de combate ao mosquito, como os que podem ser encontrados em peças publicitárias em diversas plataformas de digitais, bem como no rádio e na televisão (MINISTÉRIO DA SAÚDE, 2018) (Figura 2 com a visualização dos tipos de resíduos).

\begin{tabular}{c} 
Resíduos sólidos \\
\hline Caixa de Isopor \\
\hline Cartela de Ovos \\
\hline Embalagem transparente \\
\hline Marmitex Isopor \\
\hline Galão 20L \\
\hline Garrafa de Vidro \\
\hline Garrafa de Cerveja \\
\hline Garrafa PET \\
\hline Lata de Cerveja \\
\hline Lata de Tinta 18L \\
\hline Lata de Tinta 5L \\
\hline Pneu \\
\hline Sacola de Plástico \\
\hline Tampa de Lixo \\
\hline Vasilha Colorida
\end{tabular}

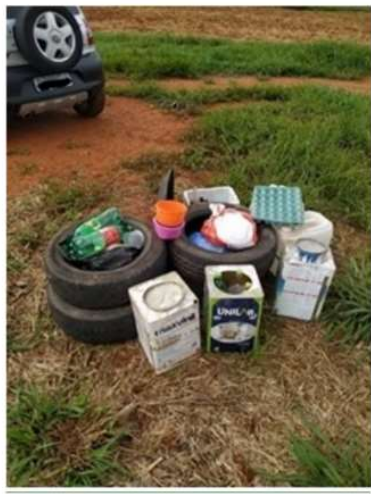

Figura 2. Relação de objetos que podem armazenar água de diferentes sítios potenciais fontes. Fonte: Autores (em 29/01/2020). Figure 2. List of objects that can store water from different potential fonts. Source: Authors (on 29/01/2020).

A identificação dos alvos ocorreu por meio de um júri representado por pessoas previamente selecionadas que 
responderam a um questionário sobre a acuidade visual relacionada à detecção de alvos (resíduos sólidos). Deve-se levar em consideração fatores como a cor do item, o contexto (ambiente de entorno imediato) onde o alvo está inserido, formato, tamanho e variação da altura do solo da imagem aérea, que implica diretamente no Ground Sample Distance (GSD) relacionado à resolução espacial da mesma. O tamanho do GSD é inversamente proporcional ao nível de detalhamento, ou seja, quanto maior o GSD (geralmente obtido em maiores alturas), menor é o nível detalhamento e quanto menor for o GSD (geralmente obtido em menores alturas), maior será o nível de detalhamento (NETO, 2016).

\subsection{Voo}

A RPA modelo Phantom 4 Pro da fabricante DJI (DJI, 2019), com câmera RGB de $20 \mathrm{Mp}$, possui adequada relação de custo $\mathrm{x}$ benefício. $\mathrm{O}$ equipamento novo custa atualmente (2021) em torno de $\mathrm{R} \$ 9.500,00$ reais $(\mathrm{U} \$ 1.720,17)$, e apresenta as seguintes especificações:

\section{Parte Física}

- Máxima altitude (nível do mar): $6000 \mathrm{~m}$

-Autonomia: 25 - 30 minutos

- Peso: $1388 \mathrm{~g}$

- Tamanho diagonal: $350 \mathrm{~mm}$

- Velocidade máxima: $72 \mathrm{~km} / \mathrm{h}$

- Sistema GNSS: GPS/GLONASS

\section{Câmera $-R G B$}

- Sensor: 1" CMOS

- Resolução: $20 \mathrm{MP}$ efetivo

- Lente: Campo de visão de $84^{\circ} 8.8 \mathrm{~mm} / 24 \mathrm{~mm}$ - f/2.8 no infinito - ISSO: 100 - 12800

- Velocidade do obturador: 8 - 1/8000 s

- Tamanho da imagem: 16:9 - 5472 x 3078

Para a obtenção das imagens dos alvos, os voos ocorreram de forma manual em 31 de janeiro de 2020, com o operador controlando o equipamento por meio do rádiocontrole, no modo estabilizado pelo sistema de geolocalização da RPA (sistema GNSS) e também por sensores anticolisão presentes no equipamento. Essa condição simulou o que um técnico de vigilância sanitária faria ao inspecionar um local com drone, com suspeita de conter focos de larvas de vetores de doença. A RPA foi direcionada para sobrevoar e pairar sobre os alvos em diferentes alturas do solo, ocorrendo o registro fotográfico de cada uma delas. A área de solo exposto não possui imagens com os alvos em $80 \mathrm{~m}, 60 \mathrm{~m}, 30 \mathrm{~m}$ e 20 metros, pois os seus alvos foram realocados para a área de vegetação seca (Figura 3).

Para encontrar o período de melhor iluminação para o voo, e assim extrair maior qualidade em termos de nitidez e contraste dos alvos nas imagens aéreas, deve-se:

- Identificar a hora de nascer e pôr do sol na cidade onde ocorrerá o voo;

- Calcular o horário de pico somando o nascer e o pôr do sol e posteriormente dividindo por dois conforme a equação 1; A equação 1 permite determinar o horário de pico para a janela de voo.

$$
\mathrm{HPL}=(\mathrm{HNS}+\mathrm{HPS}) / 2
$$

em que: HPL = Horário de Pico de Luminosidade Solar (faixa horária com menos sombra no dia); HNS: Horário do nascer do Sol em hora, minutos e segundos; HPP: Horário do pôr do Sol em hora, minutos e segundos.

\subsection{Análise dos dados}

Para aferir o quanto as imagens aéreas apoiam a identificação dos habitats de Aedes aegypti, a pesquisa considerou três variáveis: tipo de alvo, tipo de ambiente e altura de tomada da fotografia aérea. A partir disso torna-se possível sugerir parâmetros de voo com RPA mais adequados para a identificação do maior número de alvos possíveis, que representam criatórios de larvas de mosquito.

Os alvos foram dispostos em quatro áreas (Figura 4), representadas por local com vegetação alta, vegetação rasteira, solo exposto e vegetação, para realização de voos automatizados e manuais. Os mesmos locais foram sobrevoados com RPA para registro ortogonal dos alvos (Figura 5), considerando quatro níveis de altura em relação ao solo $20 \mathrm{~m}, 30 \mathrm{~m}, 60 \mathrm{~m}$ e $80 \mathrm{~m}$ (Figura 6).

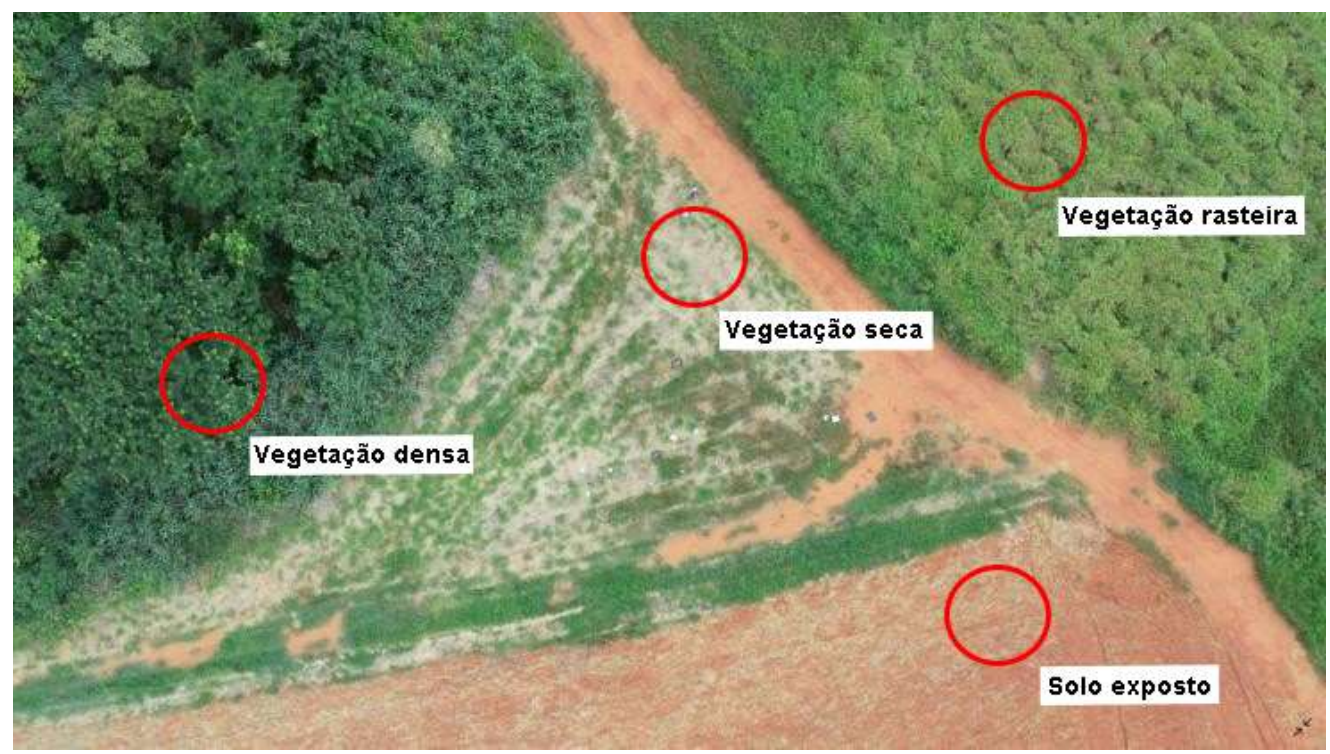

Figura 3. Mosaico de imagens aéreas extraído de RPA que operava a 30 metros do solo indicando as áreas escolhidas.

Figure 3. Mosaic of aerial images extracted from RPA that operated at 30 meters from the ground, indicating the chosen areas. 

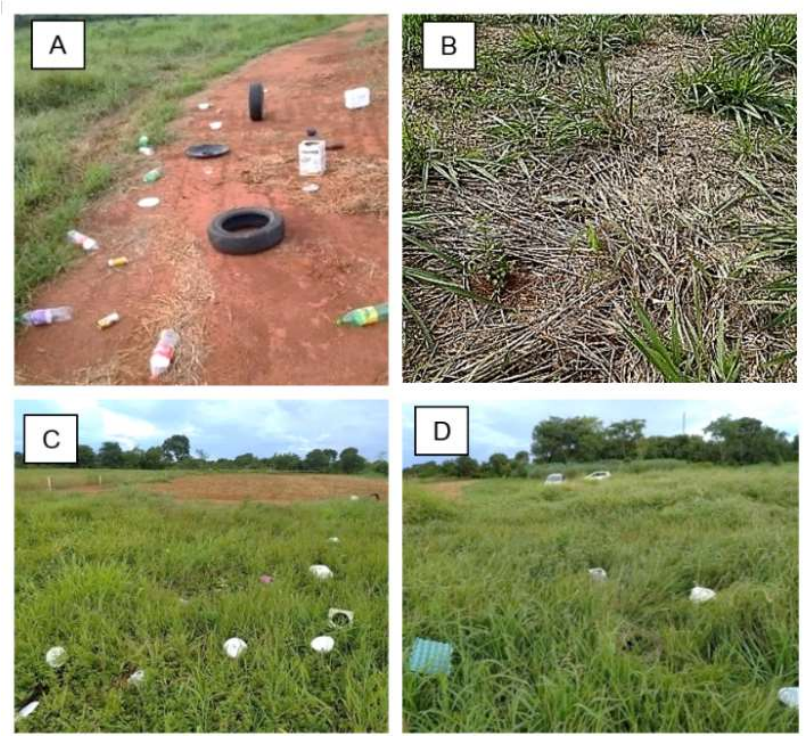

Figura 4. Disposição dos alvos na área de solo exposto (A), solo coberto com vegetação predominantemente seca (B), solo coberto com gramíneas de porte rasteiro (C) e de porte alto (D).

Figure 4. Arrangement of targets in the exposed soil area (A), soil covered with predominantly dry vegetation (B), soil covered with creeping grass $(C)$ and covered with denser and taller grass (D).
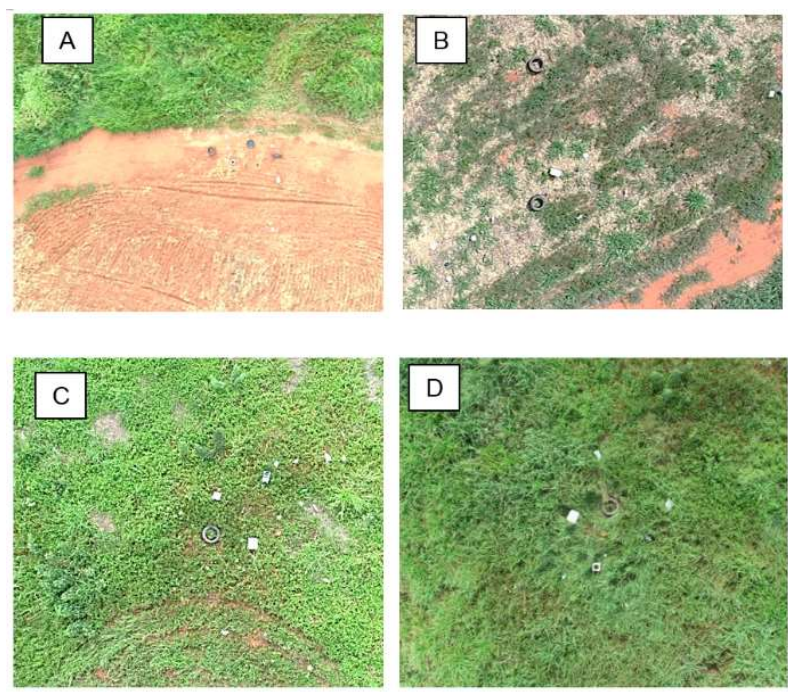

Figura 5. Disposição dos alvos a partir de vista aérea, na área de solo exposto (A), solo coberto com vegetação predominantemente seca (B), solo coberto com gramínea de porte rasteiro (C) e com gramínea de porte alto $(\mathrm{D})$.

Figure 5. Arrangement of targets from an aerial view, in the exposed soil area (A), soil covered with predominantly dry vegetation (B), soil covered with creeping grass $(\mathrm{C})$ and covered with denser and taller grass (D).
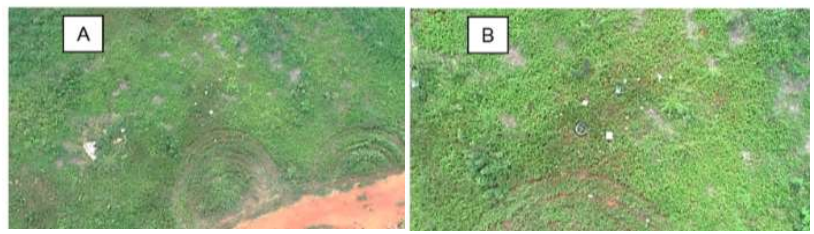

Figura 6. Disposição dos alvos em área de solo coberto com vegetação rasteira à $60(\mathrm{~A})$ e $30 \mathrm{~m}(\mathrm{~B})$.

Figure 6. Arrangement of targets in an area of ground covered with undergrowth at $60(\mathrm{~A})$ and $30 \mathrm{~m}(\mathrm{~B})$.

\subsection{Análise de júri independente}

No intuito de simular a percepção visual de um determinado número de pessoas, com relação à capacidade de identificação dos alvos por meio de imagens aéreas obtidas por RPA, houve a constituição de um grupo formado por 10 pessoas não especialistas em fotointerpretação de imagens aéreas. Esse grupo de pessoas foi denominado de júri com objetivo de informar quais eram os melhores parâmetros de registros fotográficos em termos de identificação dos alvos, utilizando apenas a percepção visual. Dos dez entrevistados $60 \%$ eram do sexo masculino e $40 \%$ do sexo feminino, com a faixa etária entre 22 e 30 anos. Todos possuem ou estão cursando ensino superior e conhecimento básico no uso de computadores.

Foi enviado ao júri um link do Google Drive (https://www.google.com/intl/pt-br/drive/about.html), arquivo na nuvem, contendo um questionário previamente elaborado para ser respondido individualmente, além dos arquivos de imagens aéreas (isoladas e ortomosaico) em formato digital. As pastas continham então uma foto da área em diferentes alturas de voo sendo elas 20, 30, 60 e 80 metros, exceto na área de solo exposto onde havia apenas as fotos de 60 e 30 metros. Os membros de júri analisam as imagens na seguinte sequência de altura de voo: $80 \mathrm{~m}, 60 \mathrm{~m}$, 30 e $20 \mathrm{~m}$.

A rotina que os membros do júri deveriam seguir era:

- Abrir o link do Google Drive;

- Identificar a pasta do Ortomosaico para se familiarizar com as áreas;

- Abrir a pasta referente ao solo exposto e abrir a imagem com a maior altura em relação ao nível do solo;

- Indicar na tabela de alvos o nome (solo exposto, solo coberto com vegetação de gramíneas predominantemente seca, solo coberto com vegetação de gramíneas rasteira inferior a $50 \mathrm{~cm}$, solo coberto com vegetação de gramíneas de porte alto - superior a $50 \mathrm{~cm}$ ), e a quantidade de todos os alvos que foi possível a identificação a essa altitude;

- Indicar o nível de dificuldade para a identificação visual desse;

- Caso não seja mais possível a identificação a essa altitude, abrir a próxima foto da mesma área, mas com uma menor altitude (exemplo se estava observando imagem registrada a $80 \mathrm{~m}$ do solo, partir para observação de imagem a $60 \mathrm{~m}$ do solo);

- Seguir fazendo o processo para a mesma área até que tenha conseguido identificar todos os alvos dessa área e variar a altura do solo, e consequentemente o nível de detalhe da imagem - GSD, de $80 \mathrm{~m}$ a $20 \mathrm{~m}$;

- Finalizando uma das áreas, deve-se abrir a nova pasta e continuar até que sejam identificados todos os alvos de todas as áreas;

- Após terminar as respostas, a planilha (Tabela 3) deveria ser enviada via e-mail para a análise dos dados;

- Alvo (nota) 1 - Alvos que, segundo o entrevistado, foram fáceis de serem identificados, essa facilidade se dá pela velocidade na identificação e quão nítido o alvo se encontrava na foto;

- Alvo (nota) 2 - Alvos que, segundo o entrevistado, tiveram dificuldade moderada para serem identificados, alvos que levaram mais tempo, que necessitaram de mais zoom ou não estavam tão nítidos na foto; 
- Alvo (nota) 3 - Alvos que, segundo o entrevistado, foram difíceis de serem identificados, com a necessidade de aplicar o zoom para ser possível a identificação.

Com esse questionário (Tabela 1), o importante era fazer o levantamento de qual altura o alvo foi encontrado com maior frequência e encontrado primeiro, caso houvesse mais de um alvo no mesmo ambiente não importava a quantidade de vezes que foi identificado e sim se pelo menos houve a identificação de um integrante do grupo de alvos.

Tabela 1. Exemplo da planilha que foi respondida pelo júri, para identificação dos alvos na área de solo exposto.

Table 1. Example of the spreadsheet that was answered by the jury, to identify the targets in the exposed soil area.

\begin{tabular}{|c|c|c|c|c|c|c|c|c|c|}
\hline \multicolumn{10}{|c|}{ TABELA DE IDENTIYICAC̣ÃO DOS ALVOS } \\
\hline TIPO DE AMBIENTE & \multirow[t]{2}{*}{ ALVO IDENTIFICADO } & \multirow[t]{2}{*}{$\begin{array}{lc}N^{n} \text { DE } \\
\text { REPETIDOS }\end{array}$} & \multirow[t]{2}{*}{ ALVOS } & \multicolumn{3}{|c|}{ ALTURA DO SOLO (m) } & \multicolumn{3}{|c|}{$\begin{array}{l}\text { NIVEL DE } \\
\text { DIFICULDADE PARA } \\
\text { IDENTIFICACÁO DO } \\
\text { ALVO } \\
\end{array}$} \\
\hline \multirow{4}{*}{ SOLO EXPOSTO } & & & & 60 & 30 & 20 & 1 & 2 & 3 \\
\hline & & & & & & & & & \\
\hline & & & & & & & & & \\
\hline & & & & & & & & & \\
\hline
\end{tabular}

\section{RESULTADOS}

$\mathrm{Na}$ área de vegetação rasteira o alvo "pneu" foi identificado a 80 metros de altura de forma unânime pelo júri. O alvo "vasilha colorida (azul)" recebeu poucas identificações e somente a 20 metros de altura. Nesse ambiente, nenhum alvo foi identificado em todas as alturas (Figura 7).

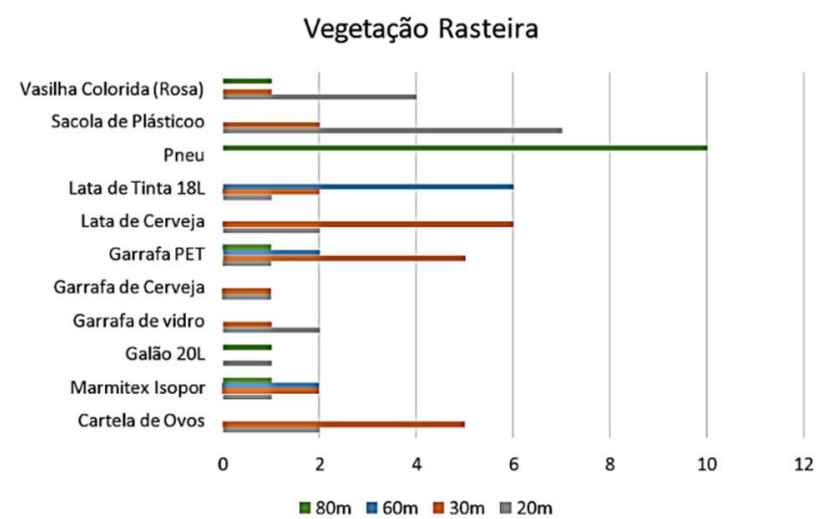

Figura 7. Alvos identificados em imagens do mesmo local variando a altura do solo para a classe solo coberto com vegetação rasteira. Figure 7. Targets identified in images from the same location varying the height of the ground for the ground covered with undergrowth class.

$\mathrm{Na}$ área de vegetação predominantemente seca o alvo "pneu" foi identificado a 80 metros de altura de forma unânime pelo júri. Os alvos "garrafa de cerveja", "garrafa de vidro" e "galão 20L" receberam o menor número de identificação. Os alvos "garrafa pet" e "marmitex isopor" foram identificados em todas as alturas nesse ambiente (Figura 8).

$\mathrm{Na}$ área de vegetação composta por gramíneas de porte alto (em média com altura maior que $50 \mathrm{~cm}$ ) o alvo "pneu" foi identificado a 80 metros de altura de forma unânime pelo júri. O alvo "Lata de tinta $5 \mathrm{~L}$ " recebeu duas ocorrências de identificação e somente a 20 metros de altura. O alvo "lata de tinta $18 \mathrm{~L}$ " foi identificado em todas as alturas nesse ambiente (Figura 9).

A Figura 10 indica o número de vezes em quem cada alvo foi identificado independente da altura, do ambiente ou do nível de dificuldade. O máximo que os alvos poderiam atingir seria o valor de 30 registros de ocorrência, que representa que esse alvo foi visualizado nos três ambientes, e todos os observadores o avistaram. Isso ocorreu com o alvo "Pneu" que foi escolhido de forma unanime nas imagens a 80 metros de altura, enquanto o alvo menos identificado foi a "Lata de tinta 5L" com apenas duas identificações, e exclusivamente à 20 metros de altura.

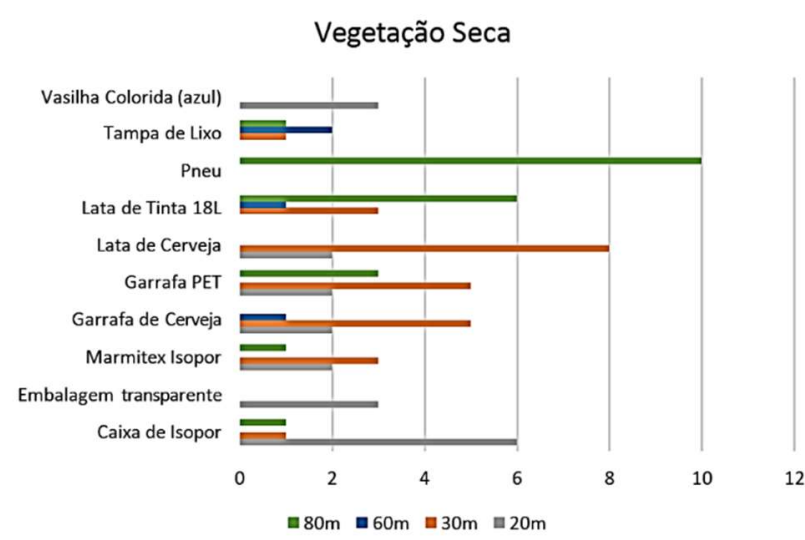

Figura 8. Alvos identificados em imagens do mesmo local variando a altura do solo para a classe solo coberto com vegetação predominantemente seca.

Figure 8. Targets identified in images from the same location varying the height of the ground for the soil class covered with predominantly dry vegetation.

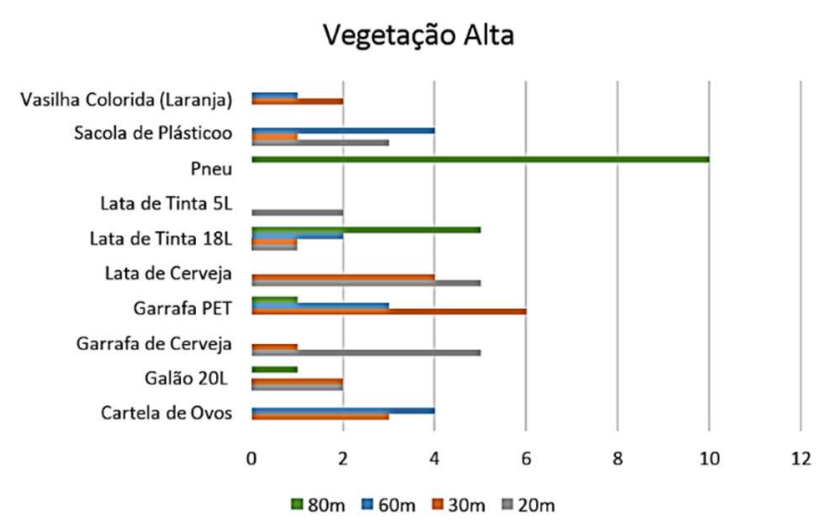

Figura 9. Alvos identificados em imagens do mesmo local variando a altura do solo para a classe solo coberto com vegetação de porte alto.

Figure 9. Targets identified in images from the same location varying the height of the ground for the soil class covered with tall vegetation. 


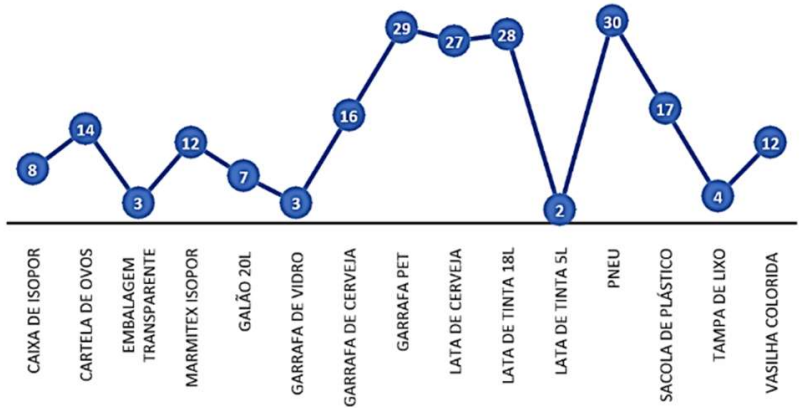

Figura 10. Registro de ocorrência em cada um dos alvos foi identificado pelo júri, independente da altura da RPA em relação ao solo, tipo de ambiente ou nível de dificuldade.

Figure 10. Occurrence record in each one of the targets was identified by the jury, regardless of the height of the RPA in relation to the ground, type of environment or level of difficulty.

Com o feedback do júri, também foi possível extrair dados relacionados à dificuldade para identificação dos alvos nessa respectiva altura em cada uma das áreas (Figura 11).

O alvo "Garrafa de Cerveja" estava presente nos três ambientes, então mesmo que tenha sido identificada muitas vezes, acabou tendo 14 registros de alvo não identificado. $\mathrm{O}$ alvo "Galão 20L" estava presente em dois ambientes, possivelmente os 13 registros de ocorrência de não identificação, seja referente a sua cor e formato, por ser todo branco era difícil encontrar detalhes que cooperassem na identificação. O alvo "Vasilha Colorida" justamente pelas suas cores, podem ter dificultado ou confundido sua identificação.

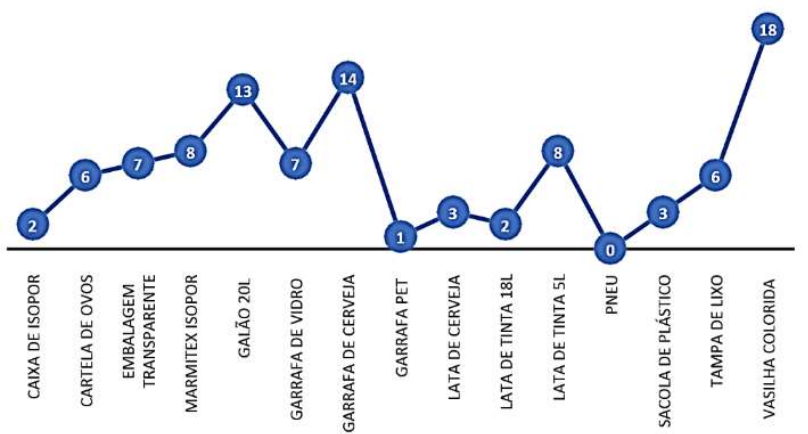

Figura 11. Registro de ocorrência dos alvos não identificados pelo júri, independente da altura da RPA em relação ao solo, tipo de ambiente ou nível de dificuldade.

Figure 11. Occurrence record of targets not identified by the jury, regardless of the height of the RPA in relation to the ground, type of environment or level of difficulty.

\section{DISCUSSÃO}

A presente técnica utilizada neste estudo é muito pouco difundida. A rotina foi desenvolvida em Rondonópolis-MT, mas pode ser replicada para outras regiões, uma vez que é necessário apenas o equipamento usado na identificação dos alvos (drone), que pode ser facilmente usado em qualquer área que se pretenda realizar voos para levantamento de criatórios de vetores. Nesse estudo foi trabalhado apenas com um sensor RBG na captura de imagens, permitindo a determinação das características locais como os tipos de vegetação e as dificuldades/facilidades de identificar os objetos (habitats) com criadouros de larvas da Dengue. No geral, o resultado mais importante deste estudo foi observar as dificuldades de identificar os alvos nos diferentes tipos de cobertura do solo, e com isso buscar novas estratégias para apresentar novas ferramentas ou métodos capazes de intervenções personalizadas no controle e vigilância da transmissão da Dengue em comunidades urbanas e em outros lugares.

Dificuldades em identificar e detectar alvos com criadouros de larvas da Dengue surgem principalmente em locais com vegetação de maior porte e frequentemente de difícil acesso, onde essa espécie pode se reproduzir com sucesso. A portabilidade das ARPs permite que os investigadores naveguem em ambientes moderadamente hostis e complexos. Este estudo além do que já foi dito, avaliou a viabilidade do uso de ARPs para gerar mapas com uma maior resolução em comparação com as disponíveis por meio de satélites, principalmente quando as imagens necessárias são específicas para uma escala local dentro de uma comunidade ou área limitada de interesse em um nível de escala microgeográfico. Estudos anteriores também propõem o uso de ARPs para mapear fatores de risco ambientais para malária zoonótica na Malásia e Filipinas (FORNACE et al., 2014), habitats de vetores em Zanzibar (HARDY et al., 2017), reprodução de Aedes aegypti (SCHENCKEL et al., 2020).

Além de imagens RGB, seria interessante também a coleta de imagens multiespectrais (NIR, RedEdge), sendo mais viável em áreas próximas de corpos hídricos ou pequenas áreas alagadas em período chuvoso, pois através de aplicações de índices multiespectrais, como por exemplo o NDVI, seria possível a distinção adequada de corpos hídricos e locais com poças de água, locais estes com grande probabilidade de criadouro de larvas de Dengue. Capturar dados várias vezes em levantamentos entomológicos com drones potencialmente forneceria as ferramentas para estudar a dinâmica de criadouros de Dengue, para determinação da prevalência da espécie em um mesmo local, promovendo a transmissão contínua. Uma vez que fossem descobertos estes habitats com uma presença perene desta espécie, estudos como esse tornar-se-iam muito importantes para uma gestão municipal antecipada no controle de proliferação da Dengue. Nesse sentido, é importante também acompanhar as modificações dos ecossistemas urbanos com drones, pois os recursos naturais frequentemente contribuem para o surgimento e disseminação de agentes de doenças infecciosas, como é o caso da Dengue. Especificamente, as mudanças no uso da terra, incluindo desmatamento, irrigação, modificação de áreas úmidas e construção de estradas, entre outros, foram identificados como principais fatores de surtos de doenças infecciosas e também podem interferir em sua dinâmica de transmissão (PATZ et al., 2004).

Nesse estudo buscamos realizar o monitoramento de controle epidemiológico do mosquito da Dengue utilizando um equipamento de menor preço de mercado (Phantom 4 Pro), por ser um equipamento barato e facilmente encontrado no mercado de tecnologias, torna-se uma ferramenta adequada para a gestão municipal, que visa o controle de gastos e ao mesmo tempo atender às demandas municipais de combate à Dengue. Entretanto, o Phatom 4 Pro fornece um único sensor RGB, o que pode limitar as possibilidades de identificação de alvos. Essa estratégia seria mais econômica à medida que o número de pesquisas com imagens RGB aumentasse. Para superar isso, alguns projetos em outras áreas estão propondo a utilização de ARPs não comerciais de baixo custo que podem ajudar a ampliar os resultados e estratégias de identificação de alvos com 
procriação de larvas de mosquito da Dengue (SASKA et al., 2012). Outra ressalva é o tempo de voo limitado do drone. O voo de uma ARP requer um certo grau de especialização, em especial para o piloto, no entanto, podem ser traçada estratégias por parte de instituições de ensino superior como as universidades federais, que poderiam realizar especializações ou cursos de curta duração na capacitação dos técnicos que irão realizar o monitoramento e busca de alvos com procriação de larvas no ambiente urbano.

\section{CONCLUSÕES}

O uso de RPA como uma ferramenta de combate ao Aedes aegypt se mostrou promissor, pois permitiu a identificação de diferentes alvos seja pela cor, formato ou tamanho, mantendo a qualidade das imagens em diferentes altitudes composições vegetais e GSD.

No ambiente solo exposto, mesmo com pouca variação de altura entre as imagens, a 60 metros houve um grande número de alvos identificados, possivelmente devido ao fato de o solo exposto oferecer um maior contraste, deixando mais evidente a grande maioria de alvos.

Nos ambientes de vegetação seca, rasteira e alta as imagens com mais identificações foram a 30 metros de altura, pois na maioria das vezes os alvos estavam relativamente mais camuflados e em alguns casos até encobertos pela vegetação do local, o que pode ter dificultado as observações em maiores alturas.

A 30 metros de altura com as imagens de $1 \mathrm{~cm}$ por pixel proporcionou o maior número de alvos identificados ao mesmo tempo que houve as melhores notas no quesito de dificuldade para identificação dos alvos, a parte negativa da escolha dessa altura é que em caso de grandes áreas onde é necessário processar as imagens e gerar ortomosaicos, haverá um grande número de fotos, resultando em mais tempo necessário para o processamento.

$O$ fato de o equipamento ser um dos RPA's mais populares no mercado, facilita replicar essa metodologia em outros lugares e difundir essa funcionalidade para a identificação, controle e combate de possíveis criatórios (alvos) do Aedes aegypti e podendo ser ampliado para outros tipos de vetores de doenças que não sejam aquelas transmitidas pelo Aedes aegypti.

Espera-se que este trabalho possa auxiliar os governos e suas políticas públicas para gestão de saúde, colaborando com agentes de saúde e de endemias.

\section{AGRADECIMENTOS}

Os autores agradecem ao Programa de Pós-Graduação em Gestão e Tecnologia Ambiental (PGTA), da Universidade Federal de Rondonópolis (UFR). Este estudo foi realizado com o apoio da Universidade Federal de Rondonópolis - UFR/MEC - Brasil.

Ao Programa de Pós-Graduação em Tecnologias Ambientais (PPGTA), da Universidade Federal de Mato Grosso do Sul (UFMS). Este estudo foi realizado com o apoio da Universidade Federal de Mato Grosso do Sul UFMS/MEC - Brasil.

A pesquisa também foi viabilizada pelo Conselho Nacional de Desenvolvimento Científico e Tecnológico (CNPq) pela bolsa de produtividade em pesquisa concedida a Normandes Matos da Silva (Processo 441975/2018-6, 305013/2018-1 e 315170/2018-2), e pela bolsa de produtividade em pesquisa concedida a A. C. Paranhos Filho (Processo CNPq 305013/2018-1).
Agradecemos à CAPES por nos fornecer acesso ao Portal de Periódicos. Por fim, gostaríamos também de agradecer à CAPES pela bolsa de doutorado concedida ao Dhonatan Diego Pessi (processo número 88887.494036/2020-00).

\section{REFERENCIAS}

ABREU, F. V. S.; MORAIS, M. M.; RIBEIRO, S. P.; EIRAS, A. E. Influence of breeding site availability on the oviposition behaviour of Aedes aegypti. Memorias do Instituto Oswaldo Cruz, Rio de Janeiro, v. 110, n. 5, p. 669-676, 2015.

ALVES, G. B. M.; LOVERDE-OLIVEIRA, S. M.; PESSI, D. D.; MARTARELLO, A. P.; VIEIRA, A.; MENDONÇA, V. M. Análise ambiental do desmatamento em área de assentamento rural no Cerrado (Mato Grosso, Brasil). Terr@Plural, v. 14, p. 1-13, e2015189, 2020.

ARAÚJO, J. G. M.; PICANÇO, A. P.; NAZARENO, J. C. Utilização de veículo aéreo não tripulado (Vant) para o monitoramento ambiental de focos do mosquito Aedes aegypti no município de Palmas, TO. Revista Gestão \& Sustentabilidade Ambiental, Florianopolis, v. 7, n. 3, p. 606 - 624, 2018. DOI: 10.19177/rgsa.v7e32018606-623

BRASIL. Ministério da Saúde. Boletim Epidemiológico Secretaria de Vigilância em Saúde Ministério da Saúde 11 Volume $51 \mathbf{N}^{\mathbf{3}}$ 34. 2020. 46P. Disponível em: https://antigo.saude.gov.br/images/pdf/2020/August/ 31/Boletim-epidemiologico-SVS-34.pdf

CARAÚBA, A. B. C.; MELO, C. S. de; SILVA, M. R. da; BARBOSA, N. R.; CORONATO, B. de O.; ANTONIO, E. R. Drones X Dengue. In: Mostra de Trabalhos Acadêmicos, IX. Anais... São Paulo: Unilus, 2015. 1p. Disponível em: http://revista.unilus.edu.br/index.php/ruep/article/vie w/606. Acesso em: 1 fev. 2020.

COSTA, M. Ocorrência do Aedes aegypti na Região Noroeste do Paraná: um estudo sobre a epidemia da Dengue em Paranavaí. 172p. Dissertação [Mestrado em Geografia] - Universidade Estadual Paulista, Presidente Prudente, 2001.

DECEA_Departamento de Controle de Espaço Aéreo. Qual a diferença entre drone, VANT e RPAS?. [S. 1.], 3 abr. 2019. Disponível em: https://ajuda.decea.gov.br/base-deconhecimento/qual-a-diferenca-entre-drone-vant-erpas/. Acesso em: 28 nov. 2019

DJI. Phantom 4 PRO. [S. 1.]. Disponível em: https://www.dji.com/br/phantom-4- pro/info. Acesso em: 28 nov. 2019.

FIOCRUZ_Fundação Oswaldo Cruz. Dengue Vírus e Vetor. Manguinhos - RJ: IOC. 2016. Disponível em: http://www.ioc.fiocruz.br/Dengue/textos/longatraje.ht ml. Acesso em: 21 out. 2019.

FORNACE, K. M.; DRAKELEY, C. J.; WILLIAM, T.; ESPINO, F.; COX, J. Mapping infectious disease landscapes: unmanned aerial vehicles and epidemiology. Trends Parasitology, v. 30, n. 11, p. 514-9, 2014. DOI: $10.1016 / j . p t .2014 .09 .001$

GOVERNO DO ESPIRITO SANTO (Brasil). Governo do Estado do Espirito Santo. Mosquito - Aedes aegypti. Espirito Santo: Secretaria de Saúde. 22 nov. 2019. Disponível em: https:/ / mosquito.saude.es.gov.br/aedesaedypti. Acesso em: 15 nov. 2019. 
GOOGLE. Google Earth website. http:/ / earth.google.com/, Data da imagem: 26/02/2019.

HARDY, A.; MAKAME, M.; CROSS, D.; MAJAMBERE, S.; MSELLEM, M. Using low-cost drones to map malaria vector habitats. Parasites and Vectors, v. 10, n. 1, p. 29, 2017. DOI: https://doi.org/10.1186/s13071-017-1973-3

IBGE_ Instituto Brasileiro de Geografia e Estatística. Disponível em: https://www.ibge.gov.br/geociencias/downloadsgeociencias.html. Acessado em: 12/01/2019.

IBGE_ Instituto Brasileiro de Geografia e Estatística. Estimativas da população residente no Brasil e Unidades da Federação com data de referência em $1^{\circ}$ de julho de 2021. Disponível em: ibge.gov.br. Acessado em: 11 de setembro de 2021

MINISTÉRIO DA SAÚDE. TODOS CONTRA O MOSQUITO. [S. 1.], 15 out. 2018. Disponível em: portalarquivos.saude.gov.br/campanhas/mosquito/. Acesso em: 24 out. 2019.

NETO, M. S. Para que serve o GSD?. [S. 1.], 15 abr. 2016. Disponível em: http://blog.droneng.com.br/gsd/. Acesso em: 31 jan. 2020.

PATZ, J. A.; DASZAK, P.; TABOR, G. M.; AGUIRRE, A. A.; PEARL, M.; EPSTEIN, J.; WOLFE, N. D.; KILPATRICK, A. M.; FOUFOPOULOS, J; MOLYNEUX, D.; BRADLEY, D. J. Unhealthy landscapes: Policy recommendations on land use change and infectious disease emergence. Environmental Health Perspectives, v. 112, n. 10, p. 1092-1098, 2004. DOI: $10.1289 /$ ehp.6877

PESSI, D. D.; LOVERDE-OLIVEIRA, S. M. Aplicação da análise geoespacial para caracterização da fragilidade de terrenos. Revista de Geografia (Recife), v. 36, n. 1, 2019.

REISKIND, M. H.; ZARRABI, A. A. Water surface area and depth determine oviposition choice in Aedes albopictus (Diptera: Culicidae). Journal of Medical Entomology, Annapolis, v. 49, n. 1, p. 71-76, 2012. DOI: https://doi.org/10.1603/ME10270

SOUZA SILVA, J.; ARIANO, Z. DE F.; SCOPEL, I. A Dengue no Brasil e as políticas de combate ao Aedes aegypti: da tentativa de erradicação às políticas de controle. Revista Brasileira de Geografia Médica e da Saúde, Uberlandia, v. 4, n. 6, p. 163-175, 2008.

SASKA, M.; KRAJNIK, T.; FAIGL, J.; VONASEK, V.; PREUCIL, L. Low cost MAV platform AR-drone in experimental verifications of methods for vision based autonomous navigation. IEEE/RSJ International Conference on Intelligent Robots and Systems, p. 712, 2012. DOI: 10.1109/IROS.2012.6386277

SCHENKEL, J.; TAELE, P.; GOLDBERG, D.; HORNEY, J.; HAMMOND, T. Identifying potential mosquito breeding grounds: assessing the efficiency of UAV technology in public health. Robotics, v. 9, e91, 2020. DOI: https://doi.org/10.3390/robotics9040091 